\title{
The Bicyclo[2.2.2]octane Motif: A Class of Saturated Group 14 Quantum Interference Based Single- molecule Insulators
}

\author{
Marc H. Garner ${ }^{1,2}$, Mads Koerstz ${ }^{1}$, Jan H. Jensen ${ }^{1}$, Gemma C. Solomon ${ }^{1,2 *}$ \\ ${ }^{1}$ Department of Chemistry and ${ }^{2}$ Nano-Science Center, University of Copenhagen, \\ Universitetsparken 5, DK-2100, Copenhagen Ø, Denmark. \\ AUTHOR INFORMATION \\ gsolomon@ nano.ku.dk
}

\section{ABSTRACT}

The electronic transmission through $\sigma$-conjugated molecules can be fully suppressed by destructive quantum interference, which makes them potential candidates for single-molecule insulators. The first molecule with clear suppression of the single-molecule conductance due to $\sigma$-interference was recently found in the form of a functionalized bicyclo[2.2.2] octasilane. Here we continue the search for potential single-molecule insulators based on saturated group 14 molecules. Using a highthroughput screening approach, we assess the electron transport properties of the bicyclo[2.2.2] octane class by systematically varying the constituent atoms between carbon, silicon, and germanium, thus exploring the full chemical space of 771 different molecules. The majority of the molecules in the bicyclo[2.2.2] octane class are found to be highly insulating molecules. Though the all-silicon molecule is a clear-cut case of $\sigma$-interference, it is not unique within its class and there are many potential molecules that we predict to be more insulating. The finding of this class of quantum interference based single-molecule insulators indicates that a broad range of highly insulating saturated group 14 molecules are likely to exist.

\section{TOC GRAPHICS}
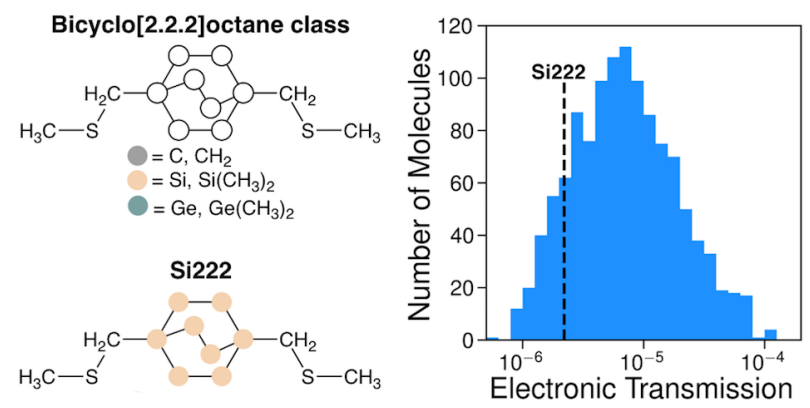
Single-molecule conductance measured at low bias in metal-molecule-metal junctions is generally a coherent electron transport process best described as a tunneling mechanism. ${ }^{1}$ The transmission through a molecule can be suppressed by destructive quantum interference, ${ }^{2}$ a phenomenon that has been demonstrated in a variety of $\pi$-conjugated systems. ${ }^{3-10}$ In principle these molecules are ideal candidates for insulating molecular units, but when transmission through the underlying $\sigma$-system is not suppressed such $\pi$-conjugated molecules cannot be more insulating than saturated molecules of equivalent length, such as alkanes. ${ }^{11-16}$

In recent work, ${ }^{16}$ we presented the first saturated molecule with destructive $\sigma$-interference in the form of a functionalized bicyclo[2.2.2] octasilane (Si222) shown in Scheme 1. With a single-molecule conductance that is much lower than saturated molecules of the same length we consider Si222 the first quantum interference based single-molecule insulator. Given that the transmission is almost completely suppressed by destructive interference, we found that removing the insulating bicyclic part of the molecule in the simulation increases the transmission by an order of magnitude. The insulating bicyclic molecular component is, in theory, a better insulator than a gap of the same size; such is the peculiar effect of destructive $\sigma$-interference. ${ }^{16}$

\section{Scheme 1.Si222.}<smiles>CSC[Si]12OO[Si](CSC)(OO1)O[Si](C)(C)O2</smiles>

Saturated $\sigma$-conjugated molecules exhibit a rich variety of electronic properties, which are welldescribed for linear silanes in the literature. ${ }^{17-21}$ The strong electronic coupling through silanes make them candidates for conducting molecular wires, ${ }^{22-25}$ and an open question is how $\sigma$-conjugation and $\sigma$-interference effects change up or down group 14 , from silicon to carbon and germanium. ${ }^{26-32}$ Wallner et al. prepared a series of mixed [2.2.2] bicyclic organosilanes ${ }^{33}$ and several mixed saturated group 14 compounds have been reported. ${ }^{34-42}$ The all-carbon bicyclo[2.2.2]octane is a commonly used insulating bridge unit, for example in the original Aviram-Ratner rectifier. ${ }^{43} \mathrm{With}$ the finding of the all-silicon counterpart as an extremely insulating moiety, the bicyclo[2.2.2] octane class of molecules is evidently a good place to start the search for single-molecule insulators. In this letter, we exhaust the full combined chemical space of saturated carbon, permethylated silicon, and permethylated germanium, as illustrated in Scheme 2, by using a high-throughput screening approach ${ }^{44}$ to asses all 771 possible molecules of the class.

\section{Scheme 2. Variation of the Bicyclo[2.2.2]octane Motif}

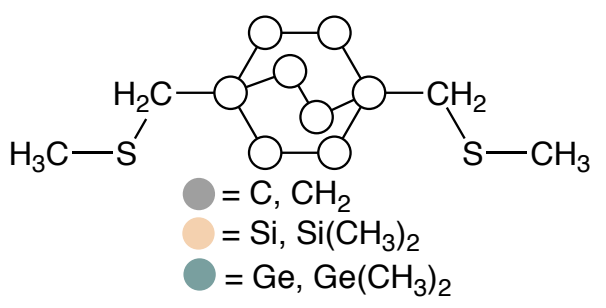


We use a custom RDKit script to generate all combinations of carbon, silicon, and germanium in the bicyclo[2.2.2] octane class as illustrated in Scheme $2 .{ }^{45}$ With three variations in 8 atoms this yields a total of $3^{8}$ possible combinations, which are reduced to 771 distinct molecules by symmetry. As described in detail in Supporting Information part I, we sample 9 conformations of each of the 771 molecules, which we optimize with GFN-xTB ${ }^{46}$ The 6939 structures are further optimized using density functional theory (DFT) with the PBE functional and a DZP basis set as implemented in the Atomic simulation environment (ASE) and GPAW. ${ }^{47-50}$ This way we obtain molecular structures of good quality; although an assessment of relative energies and thermodynamic properties may require higher level calculations.

We calculate the Landauer transmission for all 6939 structures using a custom-made transport code implemented with GPAW. As described in Supporting Information part I, this approach employs wide-band electrodes with the non-equilibrium Green's function (NEGF) approach and DFT using the PBE functional and DZP basis set. Our transport code allows for very cost-effective calculation of the transmission without compromising the quality of the electronic structure calculation; using an energy grid of 200 data-points it takes less than 10 minutes to complete the transmission calculation of Si222 on a single processor. All custom scripts are described in Supporting Information part I and are available as open-source code.

From the wide-band transmission, we select the most insulating conformations of each molecule for further analysis. The energetic positions of antiresonances in the transmission are very sensitive to changes in the electronic structure, and therefore also method dependent. Furthermore, the Fermi energy of the electrodes cannot be predicted exactly with DFT. ${ }^{51}$ For each molecule we select both the conformation with the lowest transmission at the Fermi energy and the conformation with the lowest transmission in the range from -1.5 and $+1.5 \mathrm{eV}$ relative to the Fermi energy; the latter criterion finds structures with a sharp antiresonance in the transmission somewhere in the vicinity of the Fermi energy. This procedure yields 1181 structures: 1 or 2 conformations from each of the 771 molecules. For around half of the 771 molecules, the conformation with the lowest transmission between -1.5 and $+1.5 \mathrm{eV}$ also has the lowest transmission at the Fermi energy and for those molecules only 1 conformation is selected.

We calculate the transmission of Au-molecule-Au junctions for the 1181 selected structures. Briefly, junctions are created by placing the molecule between four-atom Au pyramids placed on $5 \times$ $5 \mathrm{Au}(111)$ surfaces. These junction structures are relaxed with the Au atoms kept fixed, then the transmission is calculated using DFT and the NEGF approach as implemented in ASE and GPAW. ${ }^{48-}$ ${ }^{50,52}$ As in the wide-band calculations, we use the PBE functional and DZP basis set for all atoms, except for Au where a DZ basis set is applied. ${ }^{47}$

The transmission calculations using wide-band and Au electrodes are performed at a similar level theory, but the two types of electrodes differ. In Figure 1, the junction structures and transmissions for Si222 are shown for the two setups. Each wide-band electrode is depicted as a hydrogen molecule; it corresponds to a one-dimensional periodic chain of hydrogen atoms as detailed in supporting information part I. The Au-electrode is depicted as an Au-pyramid. Naturally, the electronic coupling of the electrodes into the sulfur atoms is quite different depending on whether it is through a hydrogen 1s orbital or an $\mathrm{Au} 6 \mathrm{~s}$ orbital. In Figure 1d it is notable that the transmission resonances are narrower using the wide-band electrodes, and consequently the transmission is generally lower. This is the consequence of the overlap between a hydrogen $1 \mathrm{~s}$ and sulfur $3 p$ orbital being smaller than that of $\mathrm{Au} 6 \mathrm{~s}$ and sulfur $3 \mathrm{p}$. This difference also seems to affect the sharpness of antiresonances in the 
transmission, which are less sharp in the Au calculation; we hypothesize this is the result of the diffuse Au orbitals coupling into multiple orbitals on sulfur and neighboring orbitals. While the transmission properties are generally preserved, we note that the antiresonance energy in Si222 changes by approximately half an $\mathrm{eV}$. This again emphasizes that it is not straightforward to find one simple selection criterion to lowering the number of conformations we screen. Ideally the transmission should be qualitatively similar to that found with the computationally expensive Au-electrodes.

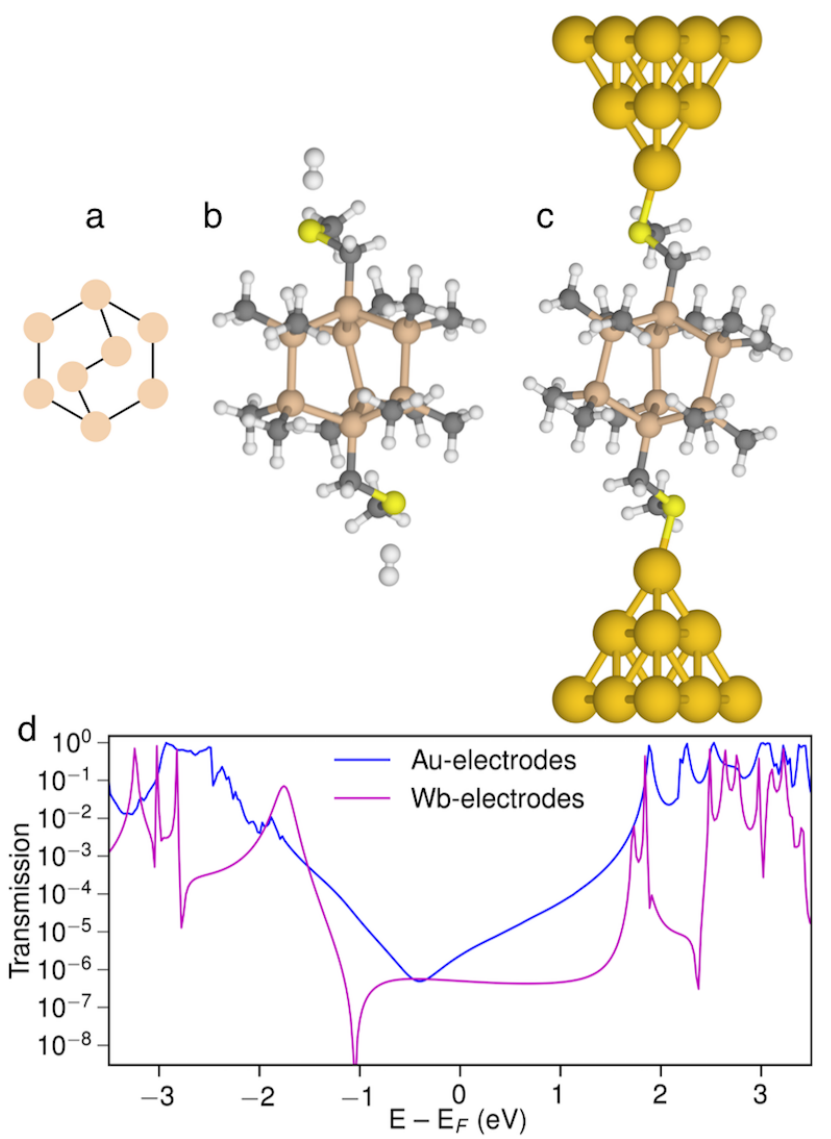

Figure 1. Structural motif (a) Wide-band junction (b), and Au-molecule-Au junction of Si222 (c). (d) Transmission calculated using the two setups for $\mathbf{S i 2 2 2}$.

Plotting the full data-set of the wide-band transmission as a histogram in Figure 2a, it is clear that there are many structures with very low transmission, i.e. around $10^{-6}$ and lower. Comparing the two data-sets with the wide-band and Au electrodes directly in Figure 2b, there are notable differences as we alluded to in the previous section. Almost every junction has lower transmission at the Fermi energy $\left(T\left(E_{F}\right)\right)$ with wide-band electrodes. For the majority, the difference is fairly systematic. However, for a significant number of junctions the transmission at the Fermi energy is several orders of magnitude lower with wide-band electrodes, and these are cases where there is a sharp antiresonance near the Fermi energy. We do not find cases where the transmission with Au electrodes becomes extremely low. This is not an error in the wide-band transmission, it is just a different junction corresponding to an experiment that cannot easily be realized - one with hydrogen atoms as electrodes. Consequently, there is promise for other realistic electrode types, or different types of experiments, where destructive interference phenomena might be even more pronounced than with Au-electrodes. 

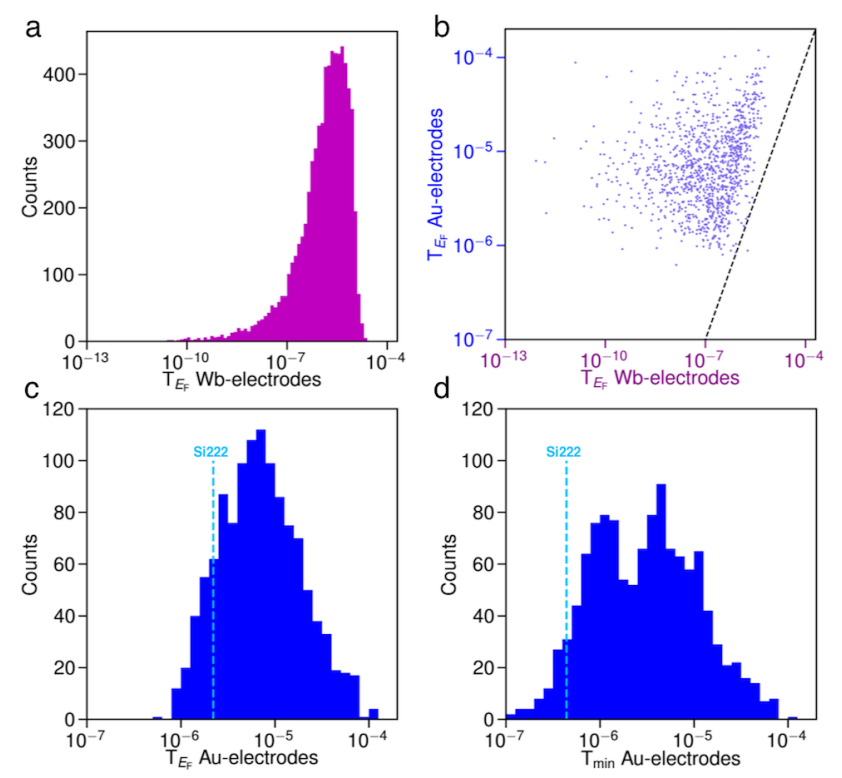

Figure 2. Histogram of $\mathrm{T}\left(\mathrm{E}_{\mathrm{F}}\right)$ for all 6939 junctions calculated using wide-band electrodes. (b) $\mathrm{T}\left(\mathrm{E}_{\mathrm{F}}\right)$ for 1181 selected junctions calculated using $\mathrm{Au}$-electrodes plotted against the $\mathrm{T}\left(\mathrm{E}_{\mathrm{F}}\right)$ calculated with wide-band electrodes. (c) Histogram of $\mathrm{T}\left(\mathrm{E}_{\mathrm{F}}\right)$ for 1181 selected junctions calculated using Auelectrodes. (d) Histogram of the minimum transmission value between -1.5 and $+1.5 \mathrm{eV}$ for 1181 selected junctions calculated using Au-electrodes.

Sorting the transmission at the Fermi energy of the 1181 Au-molecule-Au junctions in a histogram in Figure 2c, we see that the values are distributed over approximately two orders of magnitude. While the transmission of Si222 is among the low ones, Si222 only has the $146^{\text {th }}$ lowest transmission at the Fermi energy of the 771 molecules of its class. Listed in Table 1 are the top 5 most insulating molecules at the Fermi energy. The most insulating one, Comp683-6 (conformation no. 6 of molecule no. 683), has over three times lower transmission at the Fermi energy than Si222. Between Comp6836 and $\mathbf{S i 2 2 2}$, another 144 molecules of the class fall in-between.

We find it informative to also look at the lowest transmission in the range $1.5 \mathrm{eV}$ above and below the Fermi energy $\left(\mathrm{T}_{\min }\right)$, looking for a sharp antiresonance within the HOMO-LUMO gap. Shown as a histogram in Figure 2d, it is clear that there are now two subclasses of molecules. A notable fraction of molecules have lower transmission than at the Fermi energy; these are the ones with a sharp antiresonance in the transmission. Again, as listed in Table 2, Si222 performs very well among the top insulating molecules and ranks as no. 65 out of the 771 molecules of its class. Whether looking at the transmission at the Fermi energy or in a broader energy range, it is clear that though $\mathbf{S i 2 2 2}$ is an exceptional insulator, it is not a particularly special molecule within its class.

At this time, we find no clear structural pattern of the top insulators; a broad variation of motifs is represented and the top insulators consist of at least two heteroatoms. Still, it is notable that between each of Comp260-7, Comp216-4, and Comp214-8 - ranked 1, 2, and 5 in Table 2-only a single germanium atom has been changed to a silicon atom. We hypothesize that there is a balance in the electronic structure that regulates the transmission. Altering the molecular structure allows a finetuning of the $\sigma$-interference effect. Understanding this interplay between the different atoms in $\sigma$ conjugated molecules using simple electronic structure models ${ }^{20-21}$ will be an important goal for future work. 
Table 1. Molecules with lowest transmission at the Fermi energy. ${ }^{a}$ Atom color scheme is shown in Scheme 2. ${ }^{b}$ Full list is included in Supporting Information. Only the lowest conformation of each molecule is included.

\begin{tabular}{|c|c|c|c|}
\hline Ranking & Name & $\mathrm{T}\left(\mathrm{E}_{\mathrm{F}}\right)$ & Structure \\
\hline 1 & Comp683-6 & $6.2 \times 10^{-7}$ & \\
\hline 2 & Comp362-4 & $8.0 \times 10^{-7}$ & \\
\hline 3 & Comp578-8 & $8.5 \times 10^{-7}$ & \\
\hline 4 & Comp538-5 & $8.8 \times 10^{-7}$ & \\
\hline 5 & Comp763-2 & $8.8 \times 10^{-7}$ & \\
\hline 146 & $\mathrm{Si} 222$ & $2.2 \times 10^{-6}$ & \\
\hline
\end{tabular}

Table 2. Molecules with lowest transmission between $\mathbf{- 1 . 5}$ and $\mathbf{1 . 5} \mathrm{eV}$. ${ }^{\mathrm{a}}$ Atom color scheme is shown in Scheme 2. ${ }^{b}$ Full list is included in Supporting Information. Only the lowest conformation of each molecule is included.

\begin{tabular}{|c|c|c|c|}
\hline Ranking & Name & $\mathrm{T}_{\min }$ & Structure \\
\hline 1 & Comp260-7 & $1.1 \times 10^{-7}$ & \\
\hline 2 & Comp216-4 & $1.2 \times 10^{-7}$ & \\
\hline 3 & Comp731-4 & $1.3 \times 10^{-7}$ & \\
\hline 4 & Comp320-8 & $1.3 \times 10^{-7}$ & \\
\hline 5 & Comp214-8 & $1.5 \times 10^{-7}$ & \\
\hline
\end{tabular}


Let us briefly compare the transmissions of the two top candidates, Comp260-7 and Comp683-6, with Si222 as plotted in Figure 3. The transmission of Comp260-7 has an antiresonance at around $1 \mathrm{eV}$ that is much sharper than that of Si222. Despite the sharp antiresonance, Comp260-7 actually has the highest transmission of the three at the Fermi energy. The transmission of Comp683-6 is suppressed over a much broader energy range without a clear antiresonance. Though all three are very good insulators, they may be suitable for different applications. A narrow antiresonance in the transmission may be suitable for conductance suppression in a small bias range, which may facilitate conductance switching. Furthermore, a sharp antiresonance is also a criterion for high thermopower, ${ }^{53-56}$ which we reported for a derivative of Si222 ${ }^{16}$ Conversely, when the transmission is suppressed over a broad energy range, the molecule will be insulating over a larger bias-range and is likely to be less sensitive to changes in the chemical environment.

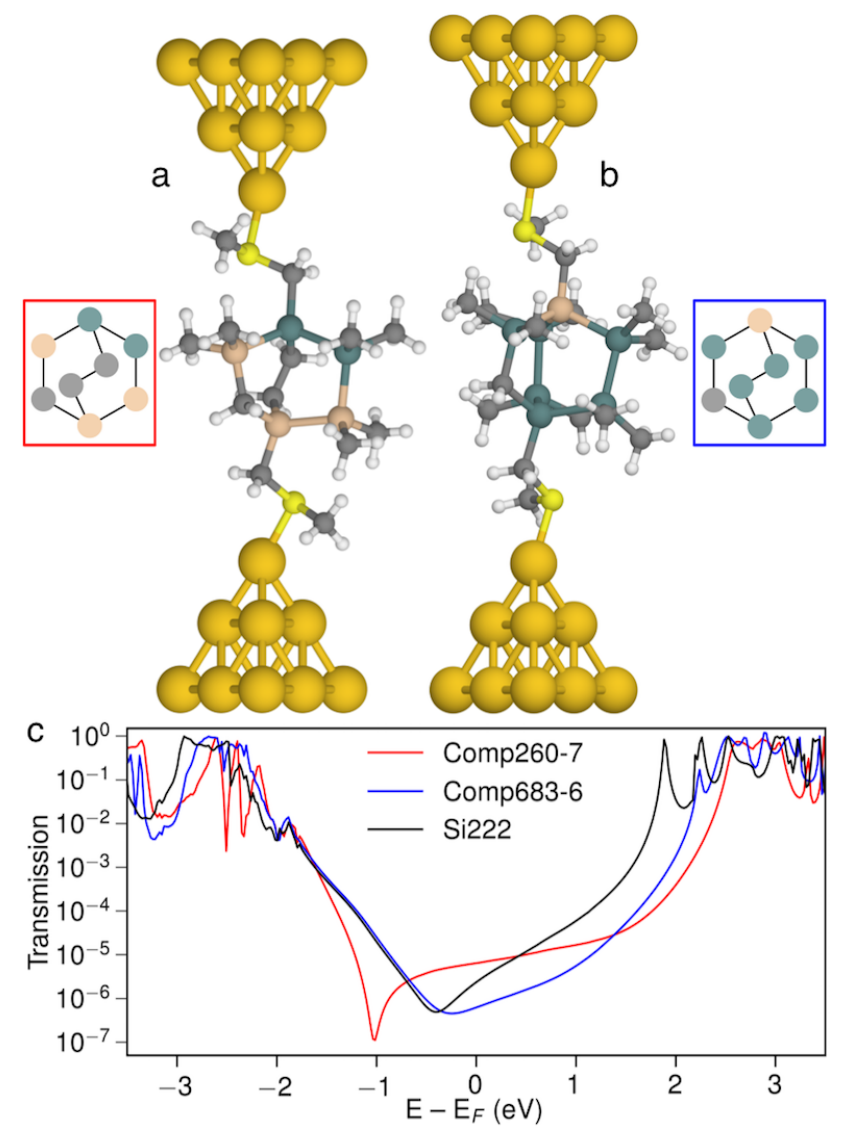

Figure 3. Top insulating Au-molecule-Au junctions and corresponding transmission, compared with Si222. (a) Optimized junction of Comp260-7. (b) Optimized junction of Comp638-6. (c) Transmission of the two junctions and $\mathbf{S i 2 2 2}$.

A special property of $\mathbf{S i 2 2 2}$ is that the insulating bicyclic unit is, in theory, more insulating than a gap the same dimensions. ${ }^{16}$ If we cut away the three silicon-bridges of the bicyclic structure, pacify the two remaining silicon atoms with hydrogen atoms and calculate the transmission for the frozen structure, the transmission is around an order of magnitude higher than that of the molecule. This 
procedure gives a measure of how strong the transmission-suppression is in the bicyclic moiety. Shown in Figure 4a-c, we perform this procedure on Comp260-7 and Comp638-6. In both cases the transmission at the Fermi energy is over an order of magnitude lower for the full molecule than for the cut junctions.

Repeating this procedure for all 1181 junctions a remarkable trend emerges. Shown in Figure 4d, the transmissions at the Fermi energy of the full junctions is plotted against that of the cut junctions. For $91 \%$ of the molecules the transmission at the Fermi energy is lower than a gap of the same dimensions; for $38 \%$ of them by more than an order of magnitude. If we instead chose the lowest transmission between -1.5 and $+1.5 \mathrm{eV}$ this trend is even more pronounced, as shown in Figure $4 \mathrm{e}$. From these results it is clear that $\mathbf{S i 2 2 2}$ is not a special molecule within its class. The majority of molecules in the bicyclo[2.2.2] octane class are exceptional single-molecule insulators.

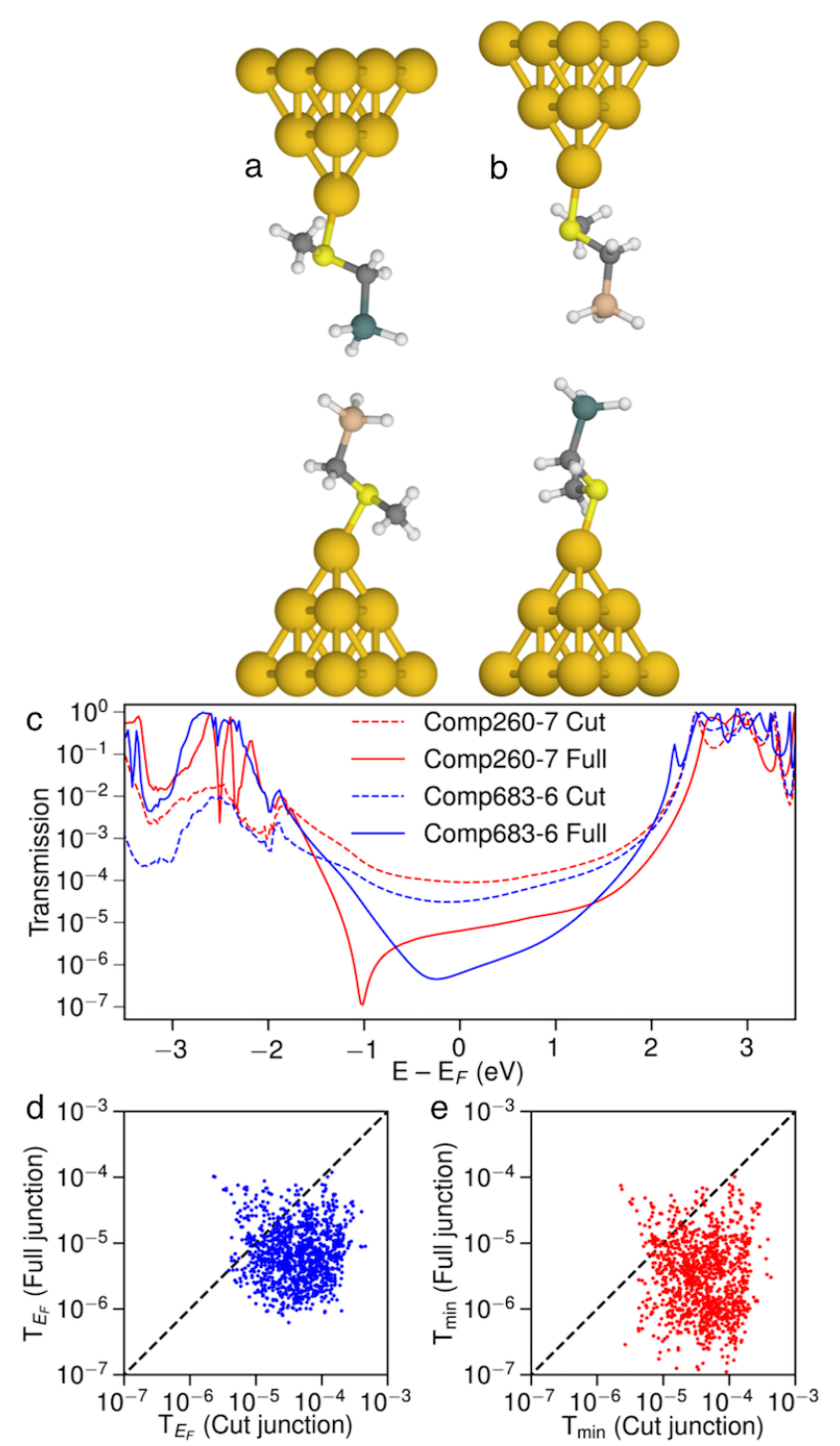

Figure 4. (a) Cut junction of Comp260-7. (b) Cut junction of Comp638-6. (c) Transmission functions of cut and full Au-molecule-Au junctions of Comp260-7 and Comp638-6. (d) Scatter plot of the transmission at the Fermi energy for the full junctions against the cut junctions for all 1182 
junctions. (e) Scatter plot of the minimum transmission between -1.5 and $1.5 \mathrm{eV}$ for the full junctions against the cut junctions for all 1182 junctions.

In summary, we have used a high-throughput approach to screen the electron transport properties of the full chemical space of the bicyclo[2.2.2] octane motif based on saturated carbon, permethylated silicon, and permethylated germanium. Like the all-silicon $\mathbf{S i 2 2 2}$, the members of this class of molecules are extremely insulating due to destructive $\sigma$-interference in the electronic transmission. We predict there are many members of the class that are even better single-molecule insulators than Si222, holding promise for future experimental efforts. While we have screened the electron transport properties of this prospective class of molecules, it is important to note that other properties remain to be screened in future efforts. The properties of single-molecule junctions depend on a number of factors, such as conformational freedom of the molecule. ${ }^{57}$ With complex cyclic and macrocyclic silicon-based molecules as realistic synthetic goals, ${ }^{58-59}$ we think high-throughput computational screening ${ }^{44}$ holds great promise for guiding future synthetic efforts by assessing large numbers of prospective molecules.

The finding of carbon, silicon, and germanium based bicyclo[2.2.2]octanes as a class of effective quantum interference based single-molecule insulators emphasizes the need for further exploration of the structure-function relationship in $\sigma$-conjugated molecules. With the perspective of inspiring new directions for research of insulating and dielectric materials, ${ }^{60-61}$ the search for single-molecule insulators continues to be an important prospect for molecular electronics.

Supporting Information. Computational details; Supplementary results.

\section{ACKNOWLEDGMENT}

We are grateful to Anders Jensen for technical contributions to our wide-band transmission code. M.H.G. and G.C.S. received funding from the Danish Council for Independent Research Natural Sciences and the Carlsberg Foundation.

\section{REFERENCES}

1. $\quad$ Su, T. A.; Neupane, M.; Steigerwald, M. L.; Venkataraman, L.; Nuckolls, C., Chemical Principles of Single-Molecule Electronics. Nat. Rev. Mater. 2016, 1, 16002.

2. Sautet, P.; Joachim, C., Electronic Interference Produced by a Benzene Embedded in a Polyacetylene Chain. Chem. Phys. Lett. 1988, 153, 511-516.

3. Mayor, M.; Weber, H. B.; Reichert, J.; Elbing, M.; von Hänisch, C.; Beckmann, D.; Fischer, M., Electric Current through a Molecular Rod-Relevance of the Position of the Anchor Groups. Angew. Chem. Int. Ed. 2003, 42, 5834-5838.

4. Taniguchi, M.; Tsutsui, M.; Mogi, R.; Sugawara, T.; Tsuji, Y.; Yoshizawa, K.; Kawai, T., Dependence of Single-Molecule Conductance on Molecule Junction Symmetry. J. Am. Chem. Soc. 2011, 133, 11426-11429.

5. Guédon, C. M.; Valkenier, H.; Markussen, T.; Thygesen, K. S.; Hummelen, J. C.; van der Molen, S. J., Observation of Quantum Interference in Molecular Charge Transport. Nat. Nanotech. 2012, 7, 305.

6. Aradhya, S. V.; Meisner, J. S.; Krikorian, M.; Ahn, S.; Parameswaran, R.; Steigerwald, M. L.; Nuckolls, C.; Venkataraman, L., Dissecting Contact Mechanics from Quantum Interference in Single-Molecule Junctions of Stilbene Derivatives. Nano Lett. 2012, 12, 1643-1647. 
7. Arroyo, C. R.; Tarkuc, S.; Frisenda, R.; Seldenthuis, J. S.; Woerde, C. H. M.; Eelkema, R.; Grozema, F. C.; van der Zant, H. S. J., Signatures of Quantum Interference Effects on Charge Transport through a Single Benzene Ring. Angew. Chem. Int. Ed. 2013, 52, 3152-3155.

8. Rabache, V.; Chaste, J.; Petit, P.; Della Rocca, M. L.; Martin, P.; Lacroix, J.-C.; McCreery, R. L.; Lafarge, P., Direct Observation of Large Quantum Interference Effect in Anthraquinone Solid-State Junctions. J. Am. Chem. Soc. 2013, 135, 10218-10221.

9. Frisenda, R.; Janssen, V. A. E. C.; Grozema, F. C.; van der Zant, H. S. J.; Renaud, N., Mechanically Controlled Quantum Interference in Individual П-Stacked Dimers. Nat. Chem. 2016, 8, 1099.

10. Zhang, Y.; Ye, G.; Soni, S.; Qiu, X.; Krijger, Theodorus L.; Jonkman, H. T.; Carlotti, M.; Sauter, E.; Zharnikov, M.; Chiechi, R. C., Controlling Destructive Quantum Interference in Tunneling Junctions Comprising Self-Assembled Monolayers Via Bond Topology and Functional Groups. Chem. Sci. 2018, 9, 4414-4423.

11. Solomon, G. C.; Andrews, D. Q.; Goldsmith, R. H.; Hansen, T.; Wasielewski, M. R.; Van Duyne, R. P.; Ratner, M. A., Quantum Interference in Acyclic Systems: Conductance of CrossConjugated Molecules. J. Am. Chem. Soc. 2008, 130, 17301-17308.

12. Andrews, D. Q.; Solomon, G. C.; Van Duyne, R. P.; Ratner, M. A., Single Molecule Electronics: Increasing Dynamic Range and Switching Speed Using Cross-Conjugated Species. J. Am. Chem. Soc. 2008, 130, 17309-17319.

13. Solomon, G. C.; Andrews, D. Q.; Van Duyne, R. P.; Ratner, M. A., Electron Transport through Conjugated Molecules: When the $\Pi$ System Only Tells Part of the Story. ChemPhysChem 2009, 10, 257-264.

14. Borges, A.; Fung, E. D.; Ng, F.; Venkataraman, L.; Solomon, G. C., Probing the Conductance of the $\Sigma$-System of Bipyridine Using Destructive Interference. J. Phys. Chem. Lett. 2016, 7, 4825-4829.

15. Li, H.; Garner, M. H.; Su, T. A.; Jensen, A.; Inkpen, M. S.; Steigerwald, M. L.; Venkataraman, L.; Solomon, G. C.; Nuckolls, C., Extreme Conductance Suppression in Molecular Siloxanes. J. Am. Chem. Soc. 2017, 139, 10212-10215.

16. Garner, M. H.; Li, H.; Chen, Y.; Su, T. A.; Shangguan, Z.; Paley, D. W.; Liu, T.; Ng, F.; Li, H.; Xiao, S., et al., Comprehensive Suppression of Single-Molecule Conductance Using Destructive $\Sigma$-Interference. Nature 2018, 558, 415-419.

17. Tsuji, H.; Terada, M.; Toshimitsu, A.; Tamao, K., $\Sigma \sigma^{*}$ Transition in Anti,Cisoid Alternating Oligosilanes: Clear-Cut Evidence for Suppression of Conjugation Effect by a Cisoid Turn. J. Am. Chem. Soc. 2003, 125, 7486-7487.

18. Fukazawa, A.; Tsuji, H.; Tamao, K., All-Anti-Octasilane: Conformation Control of Silicon Chains Using the Bicyclic Trisilane as a Building Block. J. Am. Chem. Soc. 2006, 128, 6800-6801.

19. Bande, A.; Michl, J., Conformational Dependence of $\sum$-Electron Delocalization in Linear Chains: Permethylated Oligosilanes. Chem. Eur. J. 2009, 15, 8504-8517.

20. Jovanovic, M.; Antic, D.; Rooklin, D.; Bande, A.; Michl, J., Intuitive Understanding of $\Sigma$ Delocalization in Loose and $\Sigma$ Localization in Tight Helical Conformations of an Oligosilane Chain. Chem. Asian J. 2017, 12, 1250-1263.

21. Jovanovic, M.; Michl, J., Understanding the Effect of Conformation on Hole Delocalization in Poly(Dimethylsilane). J. Am. Chem. Soc. 2018, 140, 11158-11160.

22. George, C. B.; Ratner, M. A.; Lambert, J. B., Strong Conductance Variation in Conformationally Constrained Oligosilane Tunnel Junctions. J. Phys. Chem. A 2009, 113, 38763880 .

23. Klausen, R. S.; Widawsky, J. R.; Steigerwald, M. L.; Venkataraman, L.; Nuckolls, C., Conductive Molecular Silicon. J. Am. Chem. Soc. 2012, 134, 4541-4544. 
24. Su, T. A.; Li, H.; Steigerwald, M. L.; Venkataraman, L.; Nuckolls, C., Stereoelectronic Switching in Single-Molecule Junctions. Nat. Chem. 2015, 7, 215.

25. Su, T. A.; Li, H.; Klausen, R. S.; Kim, N. T.; Neupane, M.; Leighton, J. L.; Steigerwald, M. L.; Venkataraman, L.; Nuckolls, C., Silane and Germane Molecular Electronics. Acc. Chem. Res. 2017, 50, 1088-1095.

26. Fogarty, H. A.; Casher, D. L.; Imhof, R.; Schepers, T.; Rooklin, D. W.; Michl, J., S Bonds: Electronic Structure, Photophysics, and Photochemistry of Oligosilanes. Pure Appl. Chem. 2003, 75, 999-1020.

27. Solomon, G. C.; Herrmann, C.; Hansen, T.; Mujica, V.; Ratner, M. A., Exploring Local Currents in Molecular Junctions. Nat. Chem. 2010, 2, 223.

28. Stueger, H.; Hasken, B.; Gross, U.; Fischer, R.; Torvisco Gomez, A., Synthesis and Properties of Bridgehead-Functionalized Permethylbicyclo[2.2.2]Octasilanes. Organometallics 2013, 32, 4490-4500.

29. Löfås, H.; Emanuelsson, R.; Ahuja, R.; Grigoriev, A.; Ottosson, H., Conductance through Carbosilane Cage Compounds: A Computational Investigation. J. Phys. Chem. C 2013, 117, 2169221699.

30. Emanuelsson, R.; Löfås, H.; Wallner, A.; Nauroozi, D.; Baumgartner, J.; Marschner, C.; Ahuja, R.; Ott, S.; Grigoriev, A.; Ottosson, H., Configuration- and Conformation-Dependent Electronic-Structure Variations in 1,4-Disubstituted Cyclohexanes Enabled by a Carbon-to-Silicon Exchange. Chem. Eur. J. 2014, 20, 9304-9311.

31. Su, T. A.; Li, H.; Zhang, V.; Neupane, M.; Batra, A.; Klausen, R. S.; Kumar, B.;

Steigerwald, M. L.; Venkataraman, L.; Nuckolls, C., Single-Molecule Conductance in Atomically Precise Germanium Wires. J. Am. Chem. Soc. 2015, 137, 12400-12405.

32. Su, T. A.; Li, H.; Klausen, R. S.; Widawsky, J. R.; Batra, A.; Steigerwald, M. L.;

Venkataraman, L.; Nuckolls, C., Tuning Conductance in $\Pi-\Sigma-\Pi$ Single-Molecule Wires. J. Am. Chem. Soc. 2016, 138, 7791-7795.

33. Wallner, A.; Emanuelsson, R.; Baumgartner, J.; Marschner, C.; Ottosson, H., Coupling of Disilane and Trisilane Segments through Zero, One, Two, and Three Disilanyl Bridges in Cyclic and Bicyclic Saturated Carbosilanes. Organometallics 2013, 32, 396-405.

34. Mallela, S. P.; Hill, S.; Geanangel, R. A., New Small-Ring Cyclogermanes: Syntheses and X-Ray Crystal Structures. Inorg. Chem. 1997, 36, 6247-6250.

35. Tamao, K.; Tsuji, H.; Terada, M.; Asahara, M.; Yamaguchi, S.; Toshimitsu, A., Conformation Control of Oligosilanes Based on Configurationally Constrained Bicyclic Disilane Units. Angew. Chem. Int. Ed. 2000, 39, 3287-3290.

36. Fogarty, H. A.; Tsuji, H.; David, D. E.; Ottosson, C.-H.; Ehara, M.; Nakatsuji, H.; Tamao, K.; Michl, J., Peralkylated Tetrasilanes: Conformational Dependence of the Photoelectron Spectrum. J. Phys. Chem. A 2002, 106, 2369-2373.

37. Fischer, R.; Frank, D.; Gaderbauer, W.; Kayser, C.; Mechtler, C.; Baumgartner, J.; Marschner, C., A, $\Omega$-Oligosilyl Dianions and Their Application in the Synthesis of Homo- and Heterocyclosilanes. Organometallics 2003, 22, 3723-3731.

38. Hlina, J.; Zitz, R.; Wagner, H.; Stella, F.; Baumgartner, J.; Marschner, C., $\Sigma$-Bond Electron Delocalization of Branched Oligogermanes and Germanium Containing Oligosilanes. Inorganica Chim. Acta 2014, 422, 120-133.

39. Tsuji, H.; Fogarty, H. A.; Ehara, M.; Fukuda, R.; Casher, D. L.; Tamao, K.; Nakatsuji, H.; Michl, J., Electronic Transitions in Conformationally Controlled Tetrasilanes with a Wide Range of Sisisisi Dihedral Angles. Chem. Eur. J. 2014, 20, 9431-9441.

40. Haas, M.; Leypold, M.; Schnalzer, D.; Torvisco, A.; Stueger, H., Stable Germenolates and Germenes with Exocyclic Structures. Organometallics 2015, 34, 5291-5297. 
41. Zaitsev, K. V.; Lermontova, E. K.; Churakov, A. V.; Tafeenko, V. A.; Tarasevich, B. N.; Poleshchuk, O. K.; Kharcheva, A. V.; Magdesieva, T. V.; Nikitin, O. M.; Zaitseva, G. S., et al., Compounds of Group 14 Elements with an Element-Element $(\mathrm{E}=\mathrm{Si}, \mathrm{Ge}, \mathrm{Sn})$ Bond: Effect of the Nature of the Element Atom. Organometallics 2015, 34, 2765-2774.

42. Schnalzer, D.; Haas, M.; Torvisco, A.; Stueger, H., Photochemical Reactivity of Cyclic Acylgermanes. Phosphorus, Sulfur, Silicon Relat. Elem. 2016, 191, 655-658.

43. Aviram, A.; Ratner, M. A., Molecular Rectifiers. Chem. Phys. Lett. 1974, 29, 277-283.

44. Sanchez-Lengeling, B.; Aspuru-Guzik, A., Inverse Molecular Design Using Machine Learning: Generative Models for Matter Engineering. Science 2018, 361, 360.

45. Landrum, G. Rdkit: Open-Source Cheminformatics, http://www.rdkit.org.

46. Grimme, S.; Bannwarth, C.; Shushkov, P., A Robust and Accurate Tight-Binding Quantum Chemical Method for Structures, Vibrational Frequencies, and Noncovalent Interactions of Large Molecular Systems Parametrized for All Spd-Block Elements $(\mathrm{Z}=1-86)$. J. Chem. Theory Comput. 2017, 13, 1989-2009.

47. Perdew, J. P.; Burke, K.; Ernzerhof, M., Generalized Gradient Approximation Made Simple. Phys. Rev. Lett. 1996, 77, 3865-3868.

48. Larsen, A. H.; Mortensen, J. J.; Blomqvist, J.; Castelli, I. E.; Christensen, R.; Dułak, M.; Friis, J.; Groves, M. N.; Hammer, B.; Hargus, C., et al., The Atomic Simulation Environment-a Python Library for Working with Atoms. J. Phys.: Condens. Matter 2017, 29, 273002.

49. Mortensen, J. J.; Hansen, L. B.; Jacobsen, K. W., Real-Space Grid Implementation of the Projector Augmented Wave Method. Phys. Rev. B 2005, 71, 035109.

50. Larsen, A. H.; Vanin, M.; Mortensen, J. J.; Thygesen, K. S.; Jacobsen, K. W., Localized Atomic Basis Set in the Projector Augmented Wave Method. Phys. Rev. B 2009, 80, 195112.

51. Tamblyn, I.; Darancet, P.; Quek, S. Y.; Bonev, S. A.; Neaton, J. B., Electronic Energy Level Alignment at Metal-Molecule Interfaces with a Gw Approach. Phys. Rev. B 2011, 84, 201402.

52. Chen, J.; Thygesen, K. S.; Jacobsen, K. W., Ab Initio Nonequilibrium Quantum Transport and Forces with the Real-Space Projector Augmented Wave Method. Phys. Rev. B 2012, 85, 155140 .

53. Bergfield, J. P.; Stafford, C. A., Thermoelectric Signatures of Coherent Transport in SingleMolecule Heterojunctions. Nano Lett. 2009, 9, 3072-3076.

54. Bergfield, J. P.; Solis, M. A.; Stafford, C. A., Giant Thermoelectric Effect from Transmission Supernodes. ACS Nano 2010, 4, 5314-5320.

55. Rincón-García, L.; Evangeli, C.; Rubio-Bollinger, G.; Agraït, N., Thermopower Measurements in Molecular Junctions. Chem. Soc. Rev. 2016, 45, 4285-4306.

56. Miao, R.; Xu, H.; Skripnik, M.; Cui, L.; Wang, K.; Pedersen, K. G. L.; Leijnse, M.; Pauly, F.; Wärnmark, K.; Meyhofer, E., et al., Influence of Quantum Interference on the Thermoelectric Properties of Molecular Junctions. Nano Lett. 2018, 18, 5666-5672.

57. Li, H.; Garner, M. H.; Shangguan, Z.; Zheng, Q.; Su, T. A.; Neupane, M.; Li, P.; Velian, A.; Steigerwald, M. L.; Xiao, S., et al., Conformations of Cyclopentasilane Stereoisomers Control Molecular Junction Conductance. Chem. Sci. 2016, 7, 5657-5662.

58. Wallner, A.; Hlina, J.; Konopa, T.; Wagner, H.; Baumgartner, J.; Marschner, C.; Flörke, U., Cyclic and Bicyclic Methylpolysilanes and Some Oligosilanylene-Bridged Derivatives.

Organometallics 2010, 29, 2660-2675.

59. Marro, E. A.; Press, E. M.; Siegler, M. A.; Klausen, R. S., Directional Building Blocks Determine Linear and Cyclic Silicon Architectures. J. Am. Chem. Soc. 2018, 140, 5976-5986.

60. Ha, Y.-G.; Everaerts, K.; Hersam, M. C.; Marks, T. J., Hybrid Gate Dielectric Materials for Unconventional Electronic Circuitry. Acc. Chem. Res. 2014, 47, 1019-1028. 
61. Bergfield, J. P.; Heitzer, H. M.; Van Dyck, C.; Marks, T. J.; Ratner, M. A., Harnessing Quantum Interference in Molecular Dielectric Materials. ACS Nano 2015, 9, 6412-6418. 